\title{
Right Ovarian Gonadoblastoma and Left Ovarian Dysgerminoma Metastatic to Para-Aortic Lymph Nodes, Extension to the Left Kidney in a Patient with Familial Swyer Syndrome
}

Mohammad Vakili' ${ }^{1}$ Setare Nassiri ${ }^{2 *}$, Azamsadat Mousavi ${ }^{2}$ and Hamide Gholami ${ }^{2}$

${ }^{1}$ Thorax Surgeon of Imam Hospital, Tehran University of Medical Science, Iran

${ }^{2}$ Gynecology Oncology of Imam Hospital, Tehran University of Medical Science, Iran

\begin{abstract}
Purpose: The aim of this study is to indicate a successful standard chemotherapy in metastatic dysgerminoma with extension to the kidney.

Case: The present submission is a case report of a right ovarian gonadoblastoma and a left ovarian dysgerminoma metastatic to the para-aortic lymph nodes with extension to the left kidney in a 21-year-old woman ultimately diagnosed with familial Swyer syndrome. The patient underwent bilateral salpingo-oophorectomy followed by 4 cycles of BEP and is without evidence of disease at nine-month follow-up.

Result: This is basically a report of successful administration of standard of care. Familial cases of Swyer syndrome have indeed been described. The risk of gonadoblastoma and dysgerminoma in women with Swyer syndrome is $\sim 15-30 \%$ and current practice is to perform bilateral gonadectomy, as was done in this case. In advanced stage, incompletely resected dysgerminoma, complete clinical response was $90 \%$ to BEP chemotherapy. Further, $95 \%$ of patients remained disease free with prolonged follow-up.

Conclusion: Thus, it is excellent response rates to BEP chemotherapy, it is standard of care to spare patients from high surgical morbidity and administer BEP chemotherapy instead. We recommend in such cases with extended tumoral involvement which surgery seems to be dangerous, due to large vessel invasion, we can start chemotherapy first, then re-evaluate, in presented case metastasis of dysgerminoma completely responded to chemotherapy and prevented the nephrectomy.
\end{abstract}

Keywords: Swyer syndrome; Dysgerminoma; Renal metastasis

Abbreviations: BEP: Bleomycin+Etoposide+Cisplatin; Ca 125: Cancer Antigen 125; AFP: Alpha-Fetoprotein; BHCG: Beta Human Gonadotrophin; LDH: Lactate Dehydrogenize; DSD: Disorders of Sex Development

\section{Introduction}

Swyer syndrome is a pure gonadal dysgenesis with the following specifications: primary amenorrhea, XY karyotype, female phenotype, female external genitalia and underdeveloped internal mullerian structures. These patients often come in primary amenorrhea, but they can have gonadal tumors in adults. The exact outbreak is unknown, but estimated 1 per 80000 births [1]

Swyer syndrome is caused by abnormal sexual differentiation in fetal period which leads to incomplete intra uterine masculinization and undifferentiated gonads [2] in 2006 a consultative meeting was described that instead of long and different terms such as female or male pseudo hermaphrodites, or inter sex disorders, use a single word named, DSD [3]. Swyer syndrome is composed of testosterone and mullerian inhibitor factor deficiency, lead to blocked gonadal progression [4]. Autosomal chromosomes' abnormalities cause $80 \%$ of cases while at another $20 \%$, delition in short arm of Y chromosome related to SRY, is responsible [5]. Patients with Swyer syndrome need to exact fallow up and bilateral gonadectomy as soon as possible due to the risk of malignant transformation such as dysgerminoma as soon as possible. Herein we have described a familial Swyer syndrome with gonadoblastoma in right ovary and dysgerminona in left gonad with a large Para aortic lymphadenopathy which had involved ipsilateral kidney.

\section{Case Presentation}

We have presented a 21 years old girl who came to us in Gynecology Oncology department of Imam Hospital of Iran, Tehran University of medical science, with abdominal pain which began two months ago. She had primary amenorrhea but despite the advice of the doctors, the exam and assessment were not reviewed. Her sister had infertility and primary amenorrhea with male karyotype, who was pregnant with egg donation after bilateral gonadectomy 3 years ago. Despite the suggestion of physicians about familial screening, they refused.

In physical examination, her height was $173 \mathrm{~cm}$ and her weight was $62 \mathrm{~kg}$. The axillary and pubic hair was in tanner 4 . Breast development was complete too. In abdominal exam there was a huge mobile round mass about $15 \mathrm{~cm}$ in lower abdomen to the extent of umbilicus, with brief tenderness. According to the stable condition of the patient we started a complete evaluation after admission. Given her familial history and abdominal mass, we requested fallowing assessment:

*Corresponding author: Setare Nassiri, Gynecologist Oncologist of Tehran University, Imam Hospital of Tehran, Iran, Tel: 989112556829; Fax: 982161192363 ; E-mail: setare_n99@yahoo.com

Received January 08, 2018; Accepted January 22, 2018; Published January 29, 2018

Citation: Vakili M, Nassiri S, Mousavi A, Gholami H (2018) Right Ovarian Gonadoblastoma and Left Ovarian Dysgerminoma Metastatic to Para-Aortic Lymph Nodes, Extension to the Left Kidney in a Patient with Familial Swyer Syndrome. J Blood Lymph 8: 203. doi:10.4172/2165-7831.1000203

Copyright: $\odot 2018$ Vakili M, et al. This is an open-access article distributed unde the terms of the Creative Commons Attribution License, which permits unrestricted use, distribution, and reproduction in any medium, provided the original author and source are credited. 
Citation: Vakili M, Nassiri S, Mousavi A, Gholami H (2018) Right Ovarian Gonadoblastoma and Left Ovarian Dysgerminoma Metastatic to ParaAortic Lymph Nodes, Extension to the Left Kidney in a Patient with Familial Swyer Syndrome. J Blood Lymph 8: 203. doi:10.4172/21657831.1000203

Page 2 of 3

Hormonal assay: follicle stimulating hormone (FSH): $93 \mathrm{miu} / \mathrm{mL}$ and luteinizing hormone (LH): $38 / 4 \mathrm{miu} / \mathrm{mL}$.

Estradiol: $11 \mathrm{ng} / \mathrm{mL}$, prolactin: $22 \mathrm{ng} / \mathrm{mL}$, Thyroid stimulating hormone (TSH): $1 / 4 \mathrm{miu} / \mathrm{mL}$.

Tumoral markers: CA 125: $10 \mathrm{u} / \mathrm{mL}, \mathrm{LDH}: 2572 \mathrm{u} / \mathrm{ml}$, AFP: $2 / 8 \mathrm{u} /$ $\mathrm{mL}$, BHCG: $4 \mathrm{mu} / \mathrm{mL}$.

Chromosomal study revealed a normal male chromosome complement.

Abdomino pelvic ultrasound revealed as a huge heterogeneous mass 151, 105 and $155 \mathrm{~mm}$ which filled a large part of pelvic cavity extended to abdomen part with prominent blood flow and a similar but smaller mass medial to the left kidney 110 and $71 \mathrm{~mm}$. to determine more precisely the state of the masses ,an abdomino pelvic CT scan with and without contrast was performed and suggested a heterogeneous hypo dense mass about 136 and $160 \mathrm{~mm}$ with multiple calcifications areas within the pelvic cavity and another similar mass with a certain border about 100 and $120 \mathrm{~mm}$ anterior to the left kidney seemed to be merged to the kidney with the effect of compression on large vessels (Figure 1).

According to male karyotype, familial history, external female genitalia and hyper gonadotropic hypogonadism, Swyer syndrome was diagnosed and laparotomy was planned due to malignant transformation in the gonads had not been removed in Swyer syndrome. Dysgerminoma was strongly possible because of elevated serum level of LDH.

After entrance to abdominal cavity we saw a round mobile and solid mass by left gonad origin which was resected and frozen section revealed as dysgerminoma, there was a streak ovary on the right side. On the Para aortic region there was a similar mass about $10 \mathrm{~cm}$ which could not be separated from the left kidney. so we requested Urologist oncologist to consult and he suggested, it seems to be a Para aortic lymph node which is merged to the large part of the left kidney and there is not a cleavage between them and large vessels therefore the
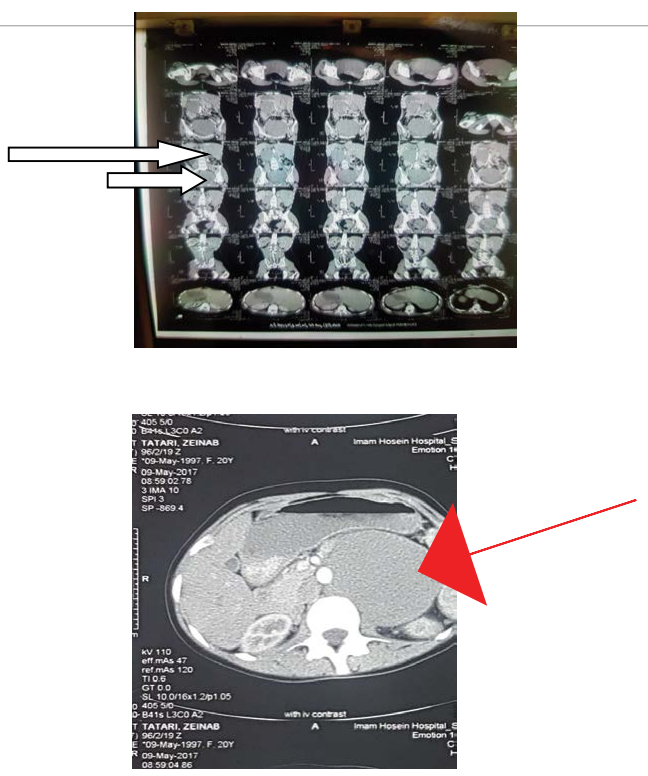

Figure 1: The long arrow shows the mass of the kidney which is below the liver and the short arrow shows pelvic mass. The red arrow shows mass of the kidney.

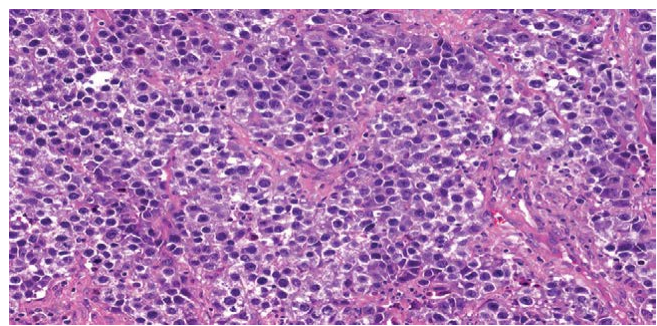

Figure 2: Grade 3 of dysgerminoma.

final comment was made during surgery that only the biopsy would be done and nephrectomy was not recognized. Intra operational pathological exam revealed as the same as left gonadal tumor. Right salpingo-oophorectomy was done and the uterus was saved to fertility preservation. There was not ascites and omental involvement but omentectomy and pelvic lymphadenectomy was performed also. She had an uncomplicated post operational period and discharged after 4 days.

The permanent pathological examination revealed as dysgerminoma, tumor greatest dimension was $18 \mathrm{~cm}$, ovarian surface was intact, and lymph vascular space invasion was evident with grade 3 of the tumor (Figure 2).

The mass consisted of a rather well defined mass which on cutting, there was a creamy whitish homogenous surface. right ovary consisted of a streak shaped gonad measuring 2.5 and $5.5 \mathrm{~cm}$ with gonadoblastoma transformation greatest diameter as $2 \mathrm{~cm}$. all of the pelvic lymph nodes were free of tumoral involvement and the biopsy of renal mass represented high grade dysgerminoma. Omentum was free of tumor

After that the history was raised in our joined committee with the presence of Gynecologist Oncologists, Uro oncologists, pathologist and radiotherapist, and the decision was finally made on chemotherapybased on this finding that dysgerminoma is a very chemo sensitive tumor and also given to the unresectable tumor on the Para aortic region invaded to the kidney. We prescribed her 4 cycles of BEP regimen included: bleomycin, etoposide, cisplatin at intervals of 21 days. Fortunately, she had a strong body and fully tolerated chemotherapy. 3 weeks after completion of chemotherapy we performed a multi slice CT scan, an interestingly but expected tumor was lost. And LDH returned to normal levels. After nine months she had no symptoms and there is no evidence of recurrence in ultrasound and in tumor marker. Then she is treated hormonal replacement therapy with estrogen and progesterone combinations and fallowed up with sonography and tumor marker every 2-3 months. Except for the sister who previously described, there was no other sister, but we suggested her to familial screening in any girl age of puberty of the family for early detection of disease.

\section{Discussion}

Pure gonadal dysgenesis of XY karyotype is named Swyer syndrome. She has female phenotype and normal but underdeveloped mullerian structures with streak gonads. In each patient with primary amenorrhea, especially with hyper gonadotropic hypogonadism karyotype assessment is mandatory [6]. Given that chromosomal defects as the cause of Swyer syndrome are known, the probability of familiarization exists. So far five cases of familial Swyer syndrome have been described and our case has been sixth [7-11].

The familial screening is still controversial because more 
Citation: Vakili M, Nassiri S, Mousavi A, Gholami H (2018) Right Ovarian Gonadoblastoma and Left Ovarian Dysgerminoma Metastatic to ParaAortic Lymph Nodes, Extension to the Left Kidney in a Patient with Familial Swyer Syndrome. J Blood Lymph 8: 203. doi:10.4172/21657831.1000203

Page 3 of 3

information is needed. But we recommended it for early detection of disease to prevent possible risk of malignant transformation with the help of gonadectomy and to start hormonal replacement therapy to maintain bone density and secondary sexual characteristics [12]. In the past the risk of malignancy did not seem to increase but today, one in every three Swyer syndrome patients gets dysgerminoma [13]. Bilateral gonadectomy in Swyer syndrome should be done as soon as possible. Uterus is often saved to fertility preservation with egg donation [14]. Dysgerminoma is often locally invasive but can be spread lymphatic or hematogen ways. Lymphatic spread is more common and occurs in retroperitoneal lymph pelvic and Para aortic lymph nodes. Distant metastases often are detected in liver, lung and bone. But renal involvement is very rare and up to now, 4 cases have been reported [15]. Our case had a tumoral tissue in Para aortic region which invaded to two thirds of the left kidney without pelvic lymph nodes involvement and without liver or lung metastases. Our patient had a unique presentation; she had something in every field. First she was a familial Swyer syndrome second, with two neoplasms in gonads, on one side had dysgerminoma and on the other side had gonadoblastoma, third she had a large renal tumoral involvement. Of course with all this difficulty, she was lucky in other field, her response to chemotherapy was complete and she did not undergo a nephrectomy that could even have been death due to large vessels invasion.

Finally, we recommended that it should be necessary to familial screening in Swyer syndrome, although more information is still needed. With early recognition, we have three goals, included: Prevention of malignant transformation in streak gonads with bilateral gonadectomy, hormone replacement therapy to avoid osteopenia, and for completion of secondary sexual characteristic. Another notable point in our patient is her complete response to chemotherapy.

\section{Conclusion}

We recommend that when faced with a tumoral involvement which surgery seems to be dangerous, due to large vessel invasion or require a nephrectomy or liver partial resection or other sites like these, we can start chemotherapy first, then re-evaluate, in presented case metastasis of dysgerminoma completely responded to chemotherapy and prevented the nephrectomy. Long fallow up is needed in such cases.

\section{Acknowledgments}

Miss Rezay of provided technical help, writing assistance and Reproductive Health Research Center, Tehran University of medical sciences provided general support.

\section{Conflict of Interest}

All authors have no conflict of interest to declare

\section{References}

1. Reddy NR, Narendra H, Sujana B, Lakshmi VVV, Radhika K (2017) Dysgerminoma in Swyer Syndrome: A Rare Case Report and Review of the Literature. Journal of Gynecologic Surgery 33.

2. Rios SD, Monteiro IC, Santos LG, Caldas NG, Chen AC, et al. (2015) A Case of Swyer Syndrome Associated with Advanced Gonadal Dysgerminoma Involving Long Survival. Case Rep Oncol 8: 179-184.

3. King TFJ, Conway GS (2014) Swyer syndrome. Curr Opin Endocrinol Diabetes Obes 21: $504-510$

4. Freitas F, Sousa C, Salazar C (2001) Male pseudohermaphroditism (in Portuguese). J Sobrage 16: 2-3.

5. Dimitri p, Cohen M, Wright $N$ (2006) Indication for familial screening and gonadectomy in patients with 46 xy gonadal dysgenesis. Int Gynecol Obstet 95: 167-168.

6. Han Y, Wang Y, Li Q, Dai S, He A, et al. (2011) Dysgerminoma in a case of 46 $X Y$ pure gonadal dysgenesis (swyer syndrome): a case report. Diagn Pathol 6: 84 .

7. Ates S, Batmaz G, Sevket O, Molla T, Dane B (2014) Familial Swyer syndrome in two sisters with undeveloped uterus. Journal of Obstetrics and Gynaecology $34: 540$.

8. Sarafoglou K, Ostrer H (2000) Clinical review 111: familial sex reversal: a review. J Clin Endocrinol Metab 85: 483-493.

9. Kempe A, Engels H, Schubert R, Meindl A, van der Ven K, et al. (2002) Familial ovarian dysgerminomas (Swyer syndrome) in females associated with $46 \mathrm{XY}$ karyotype. Gynecol Endocrinol 16: 107-111.

10. Bagci G, Bisgin A, Karauzum SB, Trak B, Luleci G (2011) Complete gonada dysgenesis 46,XY (Swyer syndrome) in two sisters and their mother's maternal aunt with a female phenotype. Fertil Steril 95: 1786.e1-3.

11. Moreira AI, Silva JC, Ferreira MS, Lanhoso A(2011) Bilateral Dysgerminoma in a Patient with a Previous Diagnosis of Swyer Syndrome. J Obstet Gynaecol Res 38: 452-454.

12. Behtash N, Zarchi MK (2007) Dysgerminoma in three patients with Swyer syndrome. World J Surg Oncol 5: 71.

13. Taylor H, Barter RH, Jacobson CB (1996) Neoplasms of dysgenetic gonads. Am J Obstet Gynecol 96: 816-823.

14. Yurekli BS, Kutbay NO, Ozen S, Karaca E, Acar K, et al. (2015) A case of Swye syndrome with gonadoblastoma and dysgerminoma. Endocrine Abstracts 37 EP1339.

15. Priyadarshi V, Sarkar K, Singh JP, Loonia R, Chakrabarty D, et al. (2013) Ovarian Dysgerminoma with Renal Metastasis: An Uncommon Phenomenon. UroToday Int J 6: art 31. 